\title{
Amygdala-Prefrontal Structural Connectivity Mediates the Relationship between Prenatal Depression and Behavior in Preschool Boys
}

\author{
Rebecca E. Hay, ${ }^{1,5}$ Jess E. Reynolds, ${ }^{2,5}$ Melody N. Grohs, ${ }^{1,5}$ Dmitrii Paniukov, ${ }^{2,4,5}$ Gerald F. Giesbrecht, ${ }^{4,5}$ \\ Nicole Letourneau, ${ }^{3,5}{ }^{\circledR}$ Deborah Dewey, ${ }^{4,5,6,7}$ and ${ }^{\circledR}$ Catherine Lebel ${ }^{2,5,6}$ \\ ${ }^{1}$ University of Calgary Cumming School of Medicine, Calgary, Alberta T2N 4N1, Canada, ${ }^{2}$ Department of Radiology, University of Calgary, Calgary, \\ Alberta T2N 4N1, Canada, ${ }^{3}$ Faculty of Nursing, University of Calgary, Calgary, Alberta T2N 4N1, Canada, ${ }^{4}$ Department of Pediatrics, University of \\ Calgary, Calgary, Alberta T2N 4N1, Canada, ${ }^{5}$ Alberta Children's Hospital Research Institute, Calgary, Alberta T3B 6A8, Canada, ${ }^{6}$ Hotchkiss Brain \\ Institute, Calgary, Alberta T2N 4N1, Canada, and ${ }^{7}$ Department of Community Health Sciences, University of Calgary, Calgary, Alberta T2N 4N1, \\ Canada
}

Prenatal depression is common, underrecognized, and undertreated. It has negative consequences on child behavior and brain development, yet the relationships among prenatal depression, child behavior, and children's brain structure remain unclear. The aim of this study was to determine whether altered brain connectivity mediates relationships between prenatal maternal depressive symptoms and child behavior. This study included 54 human mother-child pairs. Mothers completed the Edinburgh Postnatal Depression Scale during the second and third trimesters of pregnancy and 3 months postpartum. Their children had diffusion MRI at age $4.1 \pm 0.8$ years, and children's behavior was assessed using the Child Behavior Checklist within 6 months of their MRI scan. Structural brain connectivity of the amygdala, fornix, uncinate fasciculus, and cingulum was assessed using fractional anisotropy and mean diffusivity and analyzed with maternal prenatal depressive symptoms as well as child behavior. Third trimester maternal Edinburgh Postnatal Depression Scale scores were positively associated with mean diffusivity in the amygdala-frontal tract and the cingulum, controlling for postpartum depression. Externalizing behavior had a sex interaction in the amygdala-frontal pathway; weaker connectivity (lower fractional anisotropy, higher mean diffusivity) was associated with worse behavior in boys. Amygdala-frontal connectivity mediated the relationship between third trimester depressive symptoms and child externalizing behavior in males. These findings suggest that altered brain structure is a mechanism via which prenatal depressive symptoms can impact child behavior, highlighting the importance of both recognition and intervention in prenatal depression.

Key words: amygdala; brain development; depression; externalizing behavior; MRI; pregnancy

Significance Statement

Understanding how prenatal maternal depression impacts child behavior is critical for appropriately treating prenatal maternal mental health problems and improving child outcomes. Here, we show white matter changes in young children exposed to maternal prenatal depressive symptoms. Children of mothers with worse depressive symptoms had weaker white matter connectivity between areas related to emotional processing. Furthermore, connectivity between the amygdala and prefrontal cortex mediated the relationship between maternal depressive symptoms and externalizing behavior in boys, showing that altered brain structure is a possible mechanism via which maternal prenatal depression impacts children's behavior. This provides important information for understanding why children of depressed mothers may be more vulnerable to depression themselves and may help shape future guidelines on maternal prenatal care. 


\section{Introduction}

Depressive symptoms affect up to $20 \%$ of women in pregnancy (Josefsson et al., 2001; Leung and Kaplan, 2009), yet prenatal depression is underrecognized and undertreated (Leung and Kaplan, 2009; Earls et al., 2010). Prenatal depression, independent of postnatal depression and anxiety, is associated with lower child intelligence (Barker et al., 2011; Evans et al., 2012), higher infant generalized anxiety and sleep problems (Gerardin et al., 2011), increased internalizing (e.g., anxiety and depression) and externalizing (e.g., aggression and hyperactivity) behavior in preschool children (Dawson et al., 2003), and increased risk for depression at 18 years (Pearson et al., 2013). Research has also suggested that outcomes are sex-specific: male infants exposed to prenatal depression tend to have poorer motor skills, higher generalized anxiety, and sleep problems than females (Gerardin et al., 2011). Evidence indicates that effective treatment of maternal prenatal depression improves child outcomes and is associated with lower levels of internalizing behavior (Foster et al., 2008).

Child internalizing and externalizing behavioral deficits associated with prenatal maternal depression might be explained, at least in part, by alterations to underlying neurologic structure and function. The stress response is regulated by the limbic circuit, including the cingulate cortex, hippocampus, and amygdala (Rajmohan and Mohandas, 2007; Sheikh et al., 2014). Dysfunction of these brain regions is associated with hyperactivity and aggression in children (Noordermeer et al., 2016), as well as the development of depression and stress disorders in youth and adults (Drevets et al., 2008). The amygdala is particularly important for emotional processing (Bjorkquist et al., 2016) through its connections to the frontal cortex (Banks et al., 2007). Altered connectivity between the amygdala and frontal cortex is associated with disruptive behaviors (Ibrahim et al., 2019), vulnerability to depression (van Eijndhoven et al., 2009; Price and Drevets, 2010; Holmes et al., 2012; Malykhin et al., 2012), anxiety, and increased stress reactivity (Prater et al., 2013; Fowler et al., 2017).

Maternal prenatal depression may impact child behavior by altering this neurologic circuit in utero. Indeed, prenatal depression is associated with altered amygdala functional connectivity in 6-month-old infants and 4.5-year-old children (Qiu et al., 2015; Wen et al., 2017), altered right amygdala microstructure at birth (Rifkin-Graboi et al., 2013), lower frontal and parietal brain activation (Dawson et al., 2003), altered uncinate fasciculus connectivity in neonates (Lautarescu et al., 2020), lower right frontal white matter microstructure at 1-month-old (Dean et al., 2018), and significant right frontal cortical thinning in children (Sandman et al., 2015; Lebel et al., 2016).

Together, these findings suggest that maternal prenatal depression affects child behavior by impacting neurologic development. However, to our knowledge, no study has investigated whether altered white matter mediates the relationship between prenatal depression and child behavior, which would provide more concrete evidence of a mechanism. The goal of this study was to determine the relationship between prenatal depression and child brain and behavior outcomes and investigate whether relationships between maternal depression and child behavior are mediated by alterations to structural connectivity, and if these effects are sex specific.

\section{Materials and Methods}

Participants

Fifty-four mothers and their children ( 24 female; $4.1 \pm 0.8$ years at MRI scan) were enrolled from an ongoing, prospective study (Kaplan et al.,
Table 1. Demographic characteristics of mothers and children enrolled in the study $^{a}$

\begin{tabular}{lcc}
\hline & Range & Mean ( \pm SD) \\
\hline Mothers & & \\
$\quad$ Maternal age at child's birth (yr) & $26-38$ & $32.3 \pm 2.8$ \\
Postsecondary education (yr) & $0-12$ & $5.53 \pm 2.8$ \\
EPDS first trimester $(n=27)$ & $0-16$ & $4.85 \pm 3.7$ \\
EPDS second trimester $(n=47)$ & $0-16$ & $4.47 \pm 4.0$ \\
EPDS third trimester $(n=53)$ & $0-18$ & $4.83 \pm 3.5$ \\
EPDS 3 months postpartum ( $n=54)$ & $0-19$ & $4.72 \pm 4.7$ \\
Children & & \\
Sex & 24 female/30 male & \\
Gestational age at birth (wk) & $35-41.86$ & $39.3 \pm 1.4$ \\
Birth weight (g) & $2230-4150$ & $3344.9 \pm 451.8$ \\
Age at scan (yr) & $2.85-6.00$ & $4.12 \pm 0.8$ \\
CBCL Externalizing behavior T scores & $28-68$ & $44.81 \pm 9.4$ \\
CBCL Internalizing behavior T scores & $20-69$ & $44.80 \pm 9.2$ \\
\hline
\end{tabular}

${ }^{a} N=54$ unless otherwise specified. EPDS, Edinburgh postnatal depression scale; CBCL, Child behavior checklist.

2014). All participants provided informed consent, and this project was approved by the University of Calgary Health Research Ethics Board. Exclusion criteria for children included diagnosed neurologic disorders, history of head trauma, genetic disorders impacting cognitive or motor function, and contraindications to MRI. Two mothers were previously diagnosed with depression, one of whom was taking a moderate dose of a selective serotonin receptor inhibitor daily during pregnancy. Two women reported anxiety at enrollment; all women were screened. Prenatal medical comorbidities included celiac disease $(n=1)$, treated hypothyroidism $(n=5)$, and gestational diabetes $(n=5)$. For demographic information, see Table 1 .

\section{Depressive symptoms}

Maternal depressive symptoms were assessed using the Edinburgh Postnatal Depression Scale (EPDS) (Cox et al., 1987; Bergink et al., 2011). Higher scores indicate worse symptoms; scores $\geq 12$ are usually consistent with a diagnosis of major depressive disorder (MDD) (Cox et al., 1987). Women completed the EPDS up to three times in pregnancy (first trimester: $11.0 \pm 2.8$ weeks, $n=27$; second trimester: $16.8 \pm 2.2$ weeks, $n=47$; third trimester: $31.5 \pm 1.1$ weeks, $n=53)$ and at 3 months postpartum (11.5 \pm 2.8 weeks, $n=54)$. Two women $(7.4 \%)$ scored $\geq 12$ on the EPDS in the first trimester, 4 women (8.5\%) in the second trimester, 2 (3.8\%) in the third trimester, and $5(9.3 \%)$ postpartum. There were no significant associations between EPDS scores at each trimester.

\section{Behavioral measures}

Within 6 months of their MRI scan (average was $6 \pm 4 \mathrm{~d}$ before the MRI), each child's parent ( 52 completed by mothers, 2 by fathers) completed the Child Behavior Checklist (CBCL) (Rescorla, 2005), a 118-item measure of child behavior that includes composite scores for internalizing and externalizing behavior (Table 1). Two children had T scores $>60$ (considered "at risk") in internalizing, and 6 children had scores $>60$ on externalizing. No children were $>70$ (considered clinically significant) in either domain. CBCL scoring is stable over time, with high reliability for up to 1 year (Frizzo et al., 2015).

\section{Imaging}

MRI data were collected at the Alberta Children Hospital on a GE 3T MR750w (General Electric) using a 32-channel head coil. Children were watching a movie of their choice during the scan; some $(n=3)$ fell asleep during the scan. Whole-brain diffusion tensor imaging (DTI) was collected using single-shot spin echo EPI with 30 diffusion encoding gradient directions at $b=750 \mathrm{~s} / \mathrm{mm}^{2}$ and 5 images at $b=0 \mathrm{~s} / \mathrm{mm}^{2}$, $\mathrm{TR}=6750 \mathrm{~ms}, \mathrm{TE}=79 \mathrm{~ms}$, and spatial resolution of $1.6 \times 1.6 \times 2.2 \mathrm{~mm}^{3}$ (resampled on scanner to $0.78 \times 0.78 \times 2.2 \mathrm{~mm}$ ); total time was 4:03 DTI was collected as part of a larger protocol that also included T1weighted anatomic imaging, passive viewing fMRI, and arterial spin labeling (Reynolds et al., 2020). 

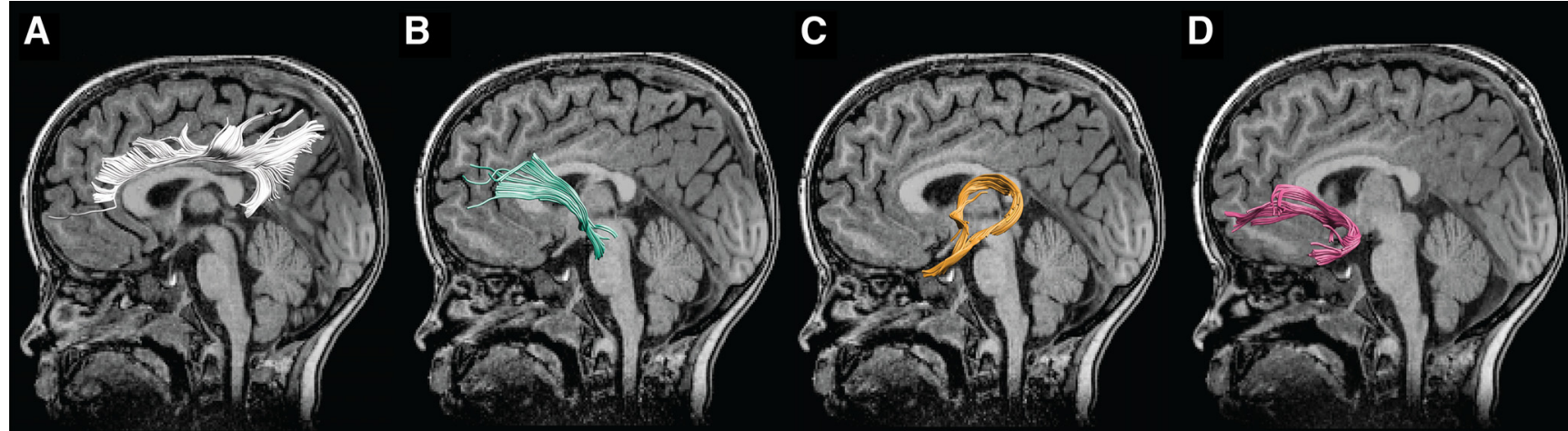

Figure 1. White matter tracts examined in this study. A, Cingulum. B, Amygdala pathway. C, Fornix. D, Uncinate fasciculus. Tracts did not overlap.

Image processing and analysis

Images were manually checked and volumes with artifacts or motion corruption were removed (Reynolds et al., 2019b). Participants with fewer than 22 volumes remaining were excluded from analysis. On average, participants had $32 \pm 4$ volumes remaining (of 35 original volumes; range 22-35). The average number of non-diffusion-weighted $(\mathrm{b}=0 \mathrm{~s} /$ $\mathrm{mm}^{2}$ ) images removed was $0.2 \pm 0.5$ (range $0-2$ ); the average number of diffusion-weighted images removed was $3.24 \pm 3.3$ (range 0-12). The number of volumes remaining was not significantly related to depressive symptoms or child behavior (all $p>0.4$ ). Preprocessing in ExploreDTI (Leemans et al., 2009) included correction for signal drift, Gibbs ringing, eddy currents, and motion. Semiautomated deterministic streamline tractography (Reynolds et al., 2019a) was used to delineate the fornix, cingulum, uncinate fasciculus, and white matter connectivity from the amygdala to the PFC (Hay et al., 2019). A representative scan (a 3.68year-old female) was identified using the tract-based spatial statistics nonlinear registration step in FSL for use as a target (Smith et al., 2006; for further details, see Reynolds et al., 2019b). All other subjects' fractional anisotropy (FA) maps were registered to this template. ROIs were drawn in the left and right hemispheres on the template and warped to fit each subject using the inverse registration parameters calculated from the previous step (Lebel et al., 2008a). Tractography was conducted in native space for each participant. Tracts were visually inspected to ensure accuracy, and spurious fibers were removed if necessary. Mean FA and mean diffusivity (MD) were extracted for each tract. Left and right tracts were examined separately for the amygdala tract, the uncinate fasciculus, and the cingulum; the fornix was analyzed as a whole, as it is a continuous tract across hemispheres. Tracts did not overlap and are shown in Figure 1.

\section{Statistical analysis}

Regression. Data met assumptions for regression (linearity, homoscedasticity, independence, normality). Brain measures (FA and MD for 7 different white matter tracts) were tested separately as dependent variables using linear regression, with maternal prenatal EPDS and an EPDSsex interaction. Based on previously established relationships with maternal depressive symptoms and/or child brain, the following potential confounding variables were also included in the regression model: child age at MRI, child sex, gestational age at birth, birth weight, maternal age, maternal postsecondary education, and postnatal EPDS. Second and third trimester EPDS symptoms were tested separately; first trimester EPDS was not assessed because of low numbers. Results were corrected for multiple comparisons using false discovery rate (FDR) based on 7 white matter tracts (left and right amygdala pathway, left and right cingulum, left and right uncinate, and fornix), 2 values each (FA and $\mathrm{MD}$ ), and 2 trimesters (second and third) for a total of 28 comparisons.

Tracts with significant (after correction) relationships with prenatal maternal depressive symptoms were then analyzed for relationships with behavioral scores. CBCL scores (Internalizing T score and Externalizing $\mathrm{T}$ score) were tested using linear regression with the brain measure and a brain-sex interaction. The same confounders as above were included in the model: child age at MRI, child sex, gestational age at birth, birth weight, maternal age, maternal postsecondary education, and postnatal EPDS. For both models, if interaction terms were not significant, they were removed from the model and the analysis was rerun. There was one outlying EPDS score; analysis was run without that person with no change in results. Similarly, the analysis was run with and without the individual on a selective serotonin receptor inhibitor, with no change in results.

Mediation. Tracts that were significantly related to both CBCL and EPDS scores were selected for mediation analysis. When sex interactions in the EPDS or CBCL regression models were significant, mediation was analyzed separately for males and females. Maternal depressive symptoms (EPDS) were entered as the independent variable (X), brain measures as the mediator $(\mathrm{M})$, and $\mathrm{CBCL}$ scores as the outcome variable $(\mathrm{Y})$. Nonparametric mediation tests were run on in-house Python using statsmodels version 0.11 .1 with percentile bootstrapping, given the validation of this model on the non-normal distribution of mediating variables (Taylor and MacKinnon, 2012).

\section{Results}

FA and MD values were in the typical range for children this age: left amygdala FA (mean: $0.42 \pm 0.01$; range: $0.36-0.46$ ), $\mathrm{MD}$ $\left(0.88 \times 10^{-3} \mathrm{~mm}^{2} / \mathrm{s} \pm 0.03 \times 10^{-3}, 0.8 \times 10^{-3}-1.0 \times 10^{-3} \mathrm{~mm}^{2} / \mathrm{s}\right)$; right amygdala FA $(0.42 \pm 0.02,0.35-0.46), \mathrm{MD}\left(0.88 \times 10^{-3}\right.$ $\mathrm{mm}^{2} / \mathrm{s} \pm 0.03 \times 10^{-3}, 0.8 \times 10^{-3}$ to $\left.1.0 \times 10^{-3} \mathrm{~mm}^{2} / \mathrm{s}\right)$; left cingulum FA $(0.42 \pm 0.02,0.36-0.48), \quad M D\left(0.87 \times 10^{-3} \mathrm{~mm}^{2} / \mathrm{s} \pm\right.$ $0.03 \times 10^{-3}, 0.8 \times 10^{-3}$ to $\left.0.9 \times 10^{-3} \mathrm{~mm}^{2} / \mathrm{s}\right)$; right cingulum $\mathrm{FA}$ $(0.40 \pm 0.03,0.35-0.44), \mathrm{MD}\left(0.86 \times 10^{-3} \mathrm{~mm}^{2} / \mathrm{s} \pm 0.03 \times 10^{-3}\right.$, $\left.0.8 \times 10^{-3}-0.9 \times 10^{-3} \mathrm{~mm}^{2} / \mathrm{s}\right)$; left uncinate fasciculus FA $(0.40 \pm$ $0.02,0.34-0.42), \mathrm{MD}\left(0.93 \times 10^{-3} \mathrm{~mm}^{2} / \mathrm{s} \pm 0.03 \times 10^{-3}, 0.9 \times\right.$ $\left.10^{-3}-1.0 \times 10^{-3} \mathrm{~mm}^{2} / \mathrm{s}\right)$; right uncinate fasciculus FA $(0.38 \pm 0.02$, $0.34-0.43), \quad \mathrm{MD}\left(0.91 \times 10^{-3} \mathrm{~mm}^{2} / \mathrm{s} \pm 0.03 \times 10^{-3}, 0.9 \times 10^{-3}\right.$ $\left.1.0 \times 10^{-3} \mathrm{~mm}^{2} / \mathrm{s}\right)$; and fornix FA $(0.40 \pm 0.02,0.33-0.40), \mathrm{MD}$ $\left(1.42 \times 10^{-3} \mathrm{~mm}^{2} / \mathrm{s} \pm 0.1 \times 10^{-3}, 1.2 \times 10^{-3}-1.8 \times 10^{-3} \mathrm{~mm}^{2} / \mathrm{s}\right)$.

\section{Cingulum}

Second trimester maternal EPDS scores had a positive main effect on left cingulum FA ( $F=4.871, p=0.033$; Table 2$)$, such that children of women with higher prenatal depressive symptoms tended to have higher FA. This result did not survive FDR correction (corrected $p$ value: $q=0.143$ ). There was a significant sex $\times$ third trimester EPDS interaction in right cingulum FA $(F=4.417, p=0.042)$, such that higher maternal depressive symptoms were associated with lower FA in males; this did not survive FDR correction $(q=0.143)$. Third trimester EPDS had a main effect on left cingulum MD $(F=10.593, p=0.002)$ and a sex $\times$ EPDS interaction $(F=5.725 p=0.021)$. Specifically, higher 
Table 2. Associations between prenatal depressive symptoms and white matter measures ${ }^{a}$

\begin{tabular}{|c|c|c|c|c|c|c|c|}
\hline \multirow[b]{2}{*}{ Cingulum } & \multicolumn{3}{|l|}{ EPDS } & \multicolumn{3}{|c|}{ EPDS $\times$ sex } & \multirow[b]{2}{*}{$R^{2}$} \\
\hline & $F$ & $p(q)$ & PES & $F$ & $p(q)$ & PES & \\
\hline \multicolumn{8}{|l|}{ Second trimester EPDS } \\
\hline Left cingulum MD & 0.203 & 0.655 & 0.005 & & & & 0.203 \\
\hline Right cingulum FA & 0.100 & 0.753 & 0.003 & & & & 0.155 \\
\hline Right cingulum MD & 0.121 & 0.729 & 0.003 & & & & 0.188 \\
\hline Left cingulum MD & 10.593 & $0.002^{b *}(0.028)$ & 0.201 & 5.725 & $0.021^{*}(0.143)$ & 0.126 & 0.483 \\
\hline Right cingulum FA & 0.161 & 0.690 & 0.004 & 4.417 & $0.042^{*}(0.143)$ & 0.095 & 0.295 \\
\hline Right cingulum MD & 5.527 & $0.023^{*}(0.143)$ & 0.114 & & & & 0.267 \\
\hline \multicolumn{8}{|l|}{ Amygdala pathway } \\
\hline \multicolumn{8}{|l|}{ Second trimester EPDS } \\
\hline Left amygdala pathway FA & 0.079 & 0.780 & 0.002 & & & & 0.294 \\
\hline Left amygdala pathway FA & 0.067 & 0.797 & 0.002 & & & & 0.349 \\
\hline Left amygdala pathway MD & 4.319 & $0.044^{*}(0.143)$ & 0.091 & & & & 0.138 \\
\hline Right amygdala pathway FA & 0.222 & 0.640 & 0.005 & & & & 0.216 \\
\hline Right amygdala pathway MD & 11.568 & $0.001^{b *}(0.028)$ & 0.212 & & & & 0.262 \\
\hline \multicolumn{8}{|l|}{ Uncinate fasciculus } \\
\hline \multicolumn{8}{|l|}{ Second trimester EPDS } \\
\hline Left uncinate fasciculus FA & 1.340 & 0.254 & 0.034 & & & & 0.391 \\
\hline Left uncinate fasciculus MD & 0.712 & 0.404 & 0.019 & 9.911 & $0.003^{b *}(0.028)$ & 0.211 & 0.406 \\
\hline Right uncinate fasciculus FA & 0.010 & 0.923 & 0.000 & & & & 0.299 \\
\hline Right uncinate fasciculus MD & 2.032 & 0.162 & 0.052 & 4.249 & $0.046^{*}(0.143)$ & 0.103 & 0.358 \\
\hline \multicolumn{8}{|l|}{ Third trimester EPDS } \\
\hline Left uncinate fasciculus FA & 0.937 & 0.339 & 0.021 & & & & 0.415 \\
\hline Left uncinate fasciculus MD & 2.392 & 0.129 & 0.052 & & & & 0.231 \\
\hline Fornix MD & 4.986 & $0.031^{*}(0.143)$ & 0.104 & & & & 0.258 \\
\hline
\end{tabular}

${ }^{a}$ Covariates were child age, sex, gestational age, birth weight, maternal age, maternal postsecondary education, and postpartum depressive symptoms. If the EPDS $\times$ sex interaction was nonsignificant, it was removed from the model, and these cells are blank. df values in EPDS2 are $(9,37)$; df values in EPDS3 are $(9,42)$ with interactions in the model. PES, Partial eta squared; EPDS, Edinburgh postnatal depression scale; FA, fractional anisotropy; $M D$, mean diffusivity.

${ }^{b}$ Results that survive FDR correction for multiple comparisons.

*Significant results.

maternal depressive symptoms were associated with higher MD in males (Fig. 2). The main effect survived FDR correction $(q=0.028)$.

Neither CBCL Externalizing nor Internalizing behavior was significantly associated with cingulum microstructure (Table 3 ).

\section{Amygdala pathway}

Third trimester maternal depressive symptoms had a positive main effect on right amygdala pathway $\mathrm{MD}(F=11.568$, $p=0.001)$ and left amygdala pathway MD $(F=4.319, p=0.044)$ (Table 2; Fig. 2). Results for the right amygdala pathway, but not the left, survived FDR correction $(q=0.028$ and $q=0.143$, respectively).

The sex $\times$ brain interactions on CBCL Externalizing behavior were significant for the right amygdala pathway $\mathrm{MD}(F=6.638$, $p=0.015)$. Higher FA and lower MD in females were associated with higher externalizing behaviors, whereas lower FA and higher MD in males were associated with higher externalizing behavior (Table 3).

\section{Uncinate fasciculus}

Second trimester maternal depressive symptoms had a significant sex interaction on left and right uncinate MD (left: $F=9.911$, $p=0.003$; right: $F=4.249, p=0.046)$ with males having a higher MD with higher maternal depressive symptoms (Table 2; Fig. 2). The positive relationship in the left uncinate, but not the right, survived multiple comparison correction $(q=0.028$ and $q=0.143$, respectively). Neither CBCL Externalizing nor Internalizing behavior was associated with uncinate microstructure (Table 3 ).

\section{Fornix}

Fornix MD was positively associated with third trimester EPDS $(F=4.986, p=0.031)$; this result did not survive multiple comparison $(q=0.143)$.

\section{Mediation analysis}

$\mathrm{MD}$ of the right amygdala significantly mediated the relationship between third trimester depressive symptoms and externalizing behavior in males (95\% CI: [0.013, 2.05] Fig. 3). 

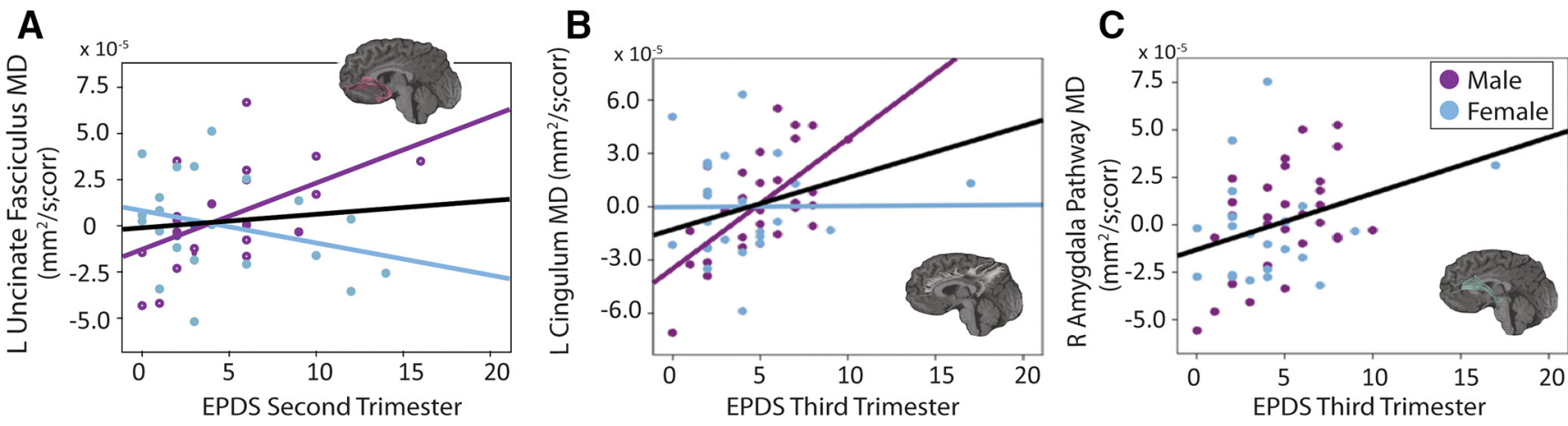

Figure 2. Relationships between white matter measures and prenatal maternal depressive symptoms. Black line indicates a main effect of Edinburgh postnatal depression scale (EPDS) scores. Lines color-coded by sex indicate an interaction between EPDS and sex. $\boldsymbol{A}$, Maternal depressive symptoms in the second trimester were positively associated with left uncinate fasciculus mean diffusivity (MD), with a significant sex interaction. Third trimester depressive symptoms were positively associated with $(\boldsymbol{B})$ left cingulum MD and (C) right amygdala pathway MD. MD values are corrected for covariates.

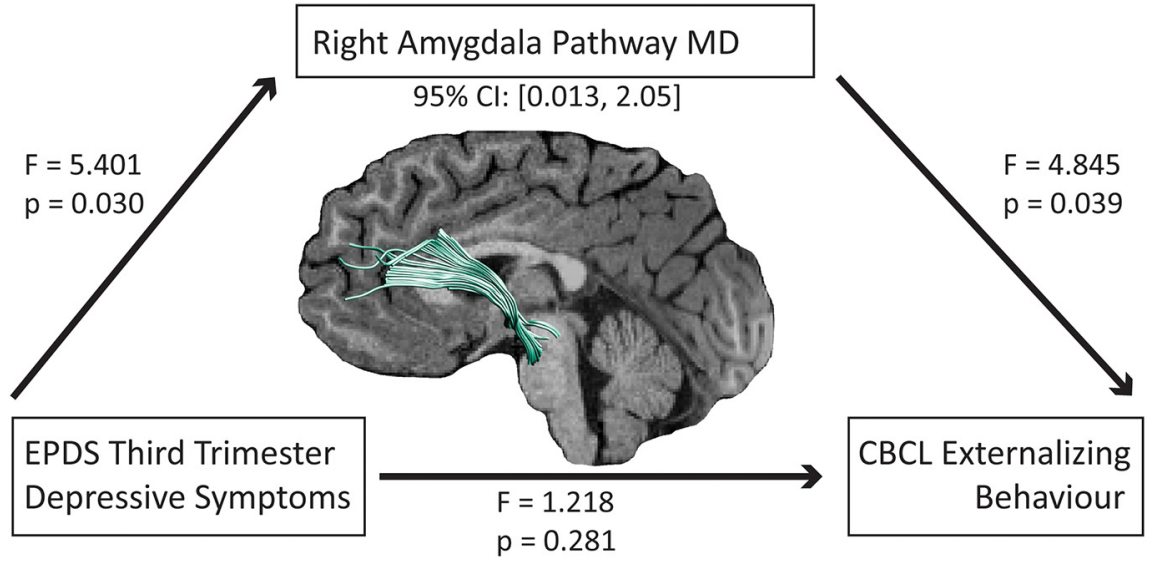

Figure 3. Mean diffusivity (MD) of the right amygdala pathway significantly mediated the relationship between third trimester depressive symptoms and externalizing behavior in males. The $95 \%$ Cl for the indirect relationship was $[0.013,2.05]$. depressive symptoms and externalizing behavior in boys. These results suggest that reduced brain connectivity within the stress network is a mechanism via which maternal depression symptoms impact children's behavior.

We found weaker structural connectivity (lower FA and higher $\mathrm{MD}$ ) in the amygdala pathway and cingulum associated with higher prenatal maternal depressive symptoms. Because FA increases and MD decreases with age (Yoshida et al., 2013), lower FA and/or higher MD values may indicate underdeveloped white matter in young children exposed to higher maternal prenatal depression symptoms, which may in turn predispose these children to dysregulated emotional states. Previous functional imaging studies have reported weaker amygdala-PFC functional

Table 3. Associations between child behavior and white matter measures in tracts that had significant relationships with maternal depressive symptoms ${ }^{a}$

\begin{tabular}{|c|c|c|c|c|c|c|c|}
\hline \multirow[b]{2}{*}{ Cingulum } & \multicolumn{3}{|l|}{ MD } & \multicolumn{3}{|c|}{$M D \times \operatorname{sex}$} & \multirow[b]{2}{*}{$R^{2}$} \\
\hline & $F$ & $p$ & PES & $F$ & $p$ & PES & \\
\hline \multicolumn{8}{|l|}{ Left cingulum } \\
\hline CBCL Internalizing & 2.393 & 0.129 & 0.052 & & & & 0.137 \\
\hline CBCL Externalizing & 0.438 & 0.512 & 0.010 & & & & 0.065 \\
\hline \multicolumn{8}{|l|}{ Amygdala pathway } \\
\hline \multicolumn{8}{|l|}{ Right amygdala pathway } \\
\hline CBCL Internalizing & 0.913 & 0.345 & 0.020 & & & & 0.109 \\
\hline CBCL Externalizing & 2.107 & 0.154 & 0.047 & 6.368 & $0.015^{*}$ & 0.129 & 0.186 \\
\hline \multicolumn{8}{|l|}{ Uncinate fasciculus } \\
\hline \multicolumn{8}{|l|}{ Left uncinate fasciculus } \\
\hline CBCL Internalizing & 0.001 & 0.979 & 0.000 & & & & 0.090 \\
\hline CBCL Externalizing & 0.057 & 0.813 & 0.001 & & & & 0.057 \\
\hline
\end{tabular}

$\overline{{ }^{a} \text { Covariates were child age, sex, gestational age, birth weight, maternal age, maternal postsecondary educa- }}$ tion, and postpartum depressive symptoms. If the interaction was nonsignificant, it was removed from the model, and these cells are blank. df values are $(9,43)$ with interactions in the model. PES, Partial eta squared; MD, mean diffusivity; CBCL, child behavior checklist.

*Significant results.

\section{Discussion}

Here, we show that prenatal maternal depressive symptoms are associated with altered limbic-prefrontal connectivity in young children. Furthermore, reduced structural connectivity between the amygdala and PFC mediated the relationship between maternal connectivity in children born to more depressed mothers (RifkinGraboi et al., 2013). Similarly, reduced functional connectivity from the amygdala to the PFC was observed in 4- to 7-year-old children exposed to early life stress, and weaker connectivity was associated with more aggression and attention difficulties (Park et al., 2018). On the other hand, Qiu et al. (2015) found greater functional connectivity from the left amygdala to frontal cortex in neonates exposed to maternal prenatal depression (Qiu et al., 2015), indicating a potentially complex picture that could depend on factors, such as child age and sex.

The cingulum was also associated with prenatal depressive symptoms, consistent with a previous study that showed increased $\mathrm{MD}$ in the cingulum of school-aged year-old children exposed to prenatal maternal depressive symptoms (El Marroun et al., 2018). The cingulum is thought to be a structure susceptible to stress (Kim et al., 2006). Lower FA has been associated with dissociation and depression in young adults exposed to early life and prenatal stress (Choi et al., 2009; Marečková et al., 2019). The hippocampus receives serotonergic transmission from the midbrain raphe via the fornix and the cingulum (Patel et al., 1996), providing a potential means by which alterations in the cingulum could disrupt mood.

Higher MD in the left uncinate fasciculus was associated with greater severity of second trimester depressive symptoms. Higher $\mathrm{MD}$, similar to lower FA, may indicate underdevelopment of this 
tract; this interestingly corresponds to other data showing reduced structural white matter connectivity of the uncincate in young adults who experienced early life stress (Hanson et al., 2015), decreased uncinate and cingulum FA in depressed adults (Bhatia et al., 2018), and decreased FA in the uncinate and cingulum of neonates exposed to prenatal anxiety (Rifkin-Graboi et al., 2015). While the uncinate was not associated with behavioral symptoms in these young children, it is possible that altered development within it may predispose children to mood disorders later on in life given data strongly suggesting uncinate involvement in MDD (Steffens et al., 2011; Carballedo et al., 2012; Bhatia et al., 2018; Deng et al., 2018).

Previous results from our laboratory in an overlapping sample suggested an accelerated pattern of development (lower diffusivity) in right frontal-temporal white matter in children exposed to greater second trimester depressive symptoms (Lebel et al., 2016). Indeed, other data have found that accelerated development may predispose children to psychiatric conditions later in life (Ono et al., 2008; Gee et al., 2013; Jalbrzikowski et al., 2017). Different results found here for third trimester may be a product of timing and brain areas examined. Indeed, in this study, we found a similar pattern of accelerated development (higher right cingulum FA in females) associated with second trimester maternal depressive symptoms, although this finding did not survive multiple comparison. These results suggest complex associations between the prenatal environment and children's brain development, where outcomes vary by timing of exposure and child age.

Altered structural connectivity similar to that observed here is associated with depression. Decreased amygdala-PFC connectivity is seen in adolescents with MDD (Cullen et al., 2014) and individuals with a familial history of depression (Luking et al., 2011). Decreased functional and structural connectivity between the amygdala and frontal cortex may indicate loss of inhibitory control and subsequent increased amygdala reactivity. This idea is supported by data showing reduced amygdala-PFC connectivity in adults associated with impaired emotional responses (Banks et al., 2007; Bjorkquist et al., 2016), anxiety and depression (Chen et al., 2008; Prater et al., 2013; Cheng et al., 2018), and increased aggression and attention problems in children (Park et al., 2018). Increased amygdala reactivity is also seen in adolescents with MDD (Perlman et al., 2012). Medications and psychotherapy that treat depression increase functional coupling between the amygdala and the striatum, thalamus, right frontal, and cingulate cortex (Chen et al., 2008), likely resulting in increased prefrontal inhibition and regulation of amygdala responsivity. It is important to note that, although data consistently show altered amygdala-PFC connectivity associated with mood and anxiety disorders, it is unclear whether that dysfunction is because of hypo- or hyper-connectivity (Jalbrzikowski et al., 2017). Our results support the theory of weaker top-down amygdala inhibition in children who experienced higher prenatal maternal depressive symptoms, providing evidence of a structural basis for previously observed deficits in functional connectivity.

Structural connectivity of the amygdala pathway mediated the relationship between maternal prenatal depressive symptoms and externalizing behavior in boys. Children whose mothers experienced prenatal depression consistently have higher externalizing symptoms (Oberlander et al., 2007), and thus alterations to amygdala-PFC structural connectivity may be a mechanism via which maternal depression impacts children's behavior (Pearson et al., 2013). Furthermore, this altered connectivity may increase children's risk for later mental health difficulties, as higher childhood externalizing symptoms are associated with later risk of suicidality, substance use, and psychopathology in adolescence and adulthood (Holtmann et al., 2011; Bellani et al., 2012). While this is the first study to show a mediating role of white matter connectivity, previous studies have shown a mediating role for gray matter in school-aged children. Right prefrontal cortical thickness mediates the relationship between maternal prenatal depressive symptoms and externalizing behavior in school-aged children (Sandman et al., 2015); in an overlapping sample, frontal cortical thickness in children mediated the relationship between prenatal maternal stress and adolescent depressive symptoms (Davis et al., 2020). Another study showed that amygdala volumes mediate the effect of prenatal maternal stress on boys' externalizing behavior at age 11 years (Jones et al., 2019), supporting the idea of sex-specific mediation effects on behavior.

The sexual differentiation suggests that boys are more vulnerable to maternal depressive symptoms. Some studies show that males experience more prominent age-related gray (De Bellis et al., 2001) and white matter changes compared with females (Simmonds et al., 2014; Reynolds et al., 2019b), although other studies suggest similar development trajectories across sexes (Lebel et al., 2008b; Muftuler et al., 2012). More rapid development in males may underlie increased susceptibility to environmental factors, such as prenatal depression. In childhood, males have a higher prevalence of depression (Merikangas et al., 2009), and male but not female children of postnatally depressed mothers have poorer cognitive function (Murray et al., 2010). Male infants exposed to prenatal depression have lower motor skills, higher generalized anxiety, and sleep problems (Gerardin et al., 2011). Thus, our data highlight that it may be underlying brain vulnerability in males that predisposes them to behavior problems. Of note, previous data in adults showed no moderating effect of sex on the relationship between prenatal stress during the first half of pregnancy and FA of the cingulum (Marečková et al., 2019). However, differences in exposure periods and the age of the offspring may account for the contrasting results.

The mechanisms through which prenatal maternal depression influences child brain structure are not well understood. Theories include dysregulation of the hypothalamic-pituitary-adrenal (HPA) axis and increased cortisol during pregnancy, genetic heritability of mood disorders, epigenetic modification of preexisting genes, nutrition, and inflammation (Weinstock, 2005). Maternal stress and depression during pregnancy increase cortisol and corticotropin releasing hormone, which initiates signaling in the HPA axis and can cross the placenta (Weinstock, 2005). Depression is associated with HPA axis dysregulation, with increased and prolonged stress responses and higher circulating glucocorticoids (Wong et al., 2000), which activate receptors in the child's brain and induce epigenetic changes (Weinstock, 2008). Animal research shows that even brief stress induces structural remodeling of the PFC, increasing emotional dysregulation and reducing fear extinction (Holmes and Wellman, 2009). Indeed, early life stress and HPA-axis function predict later psychopathology, potentially through connections with the amygdala-ventromedial PFC (Burghy et al., 2012); thus, prenatal HPA axis dysregulation and cortisol exposure may induce similar changes in amygdala projections to the PFC. Additionally, high stress in late pregnancy is associated with reduced uterine blood flow, suggesting that fetuses experience periods of hypoxia (Weinstock, 2008) that may impact brain development. MDD is highly heritable (Lohoff, 2010), and preexisting genetic vulnerability likely plays an important role in child vulnerability. Poor nutrition during pregnancy increases the risk of perinatal depression and poor child cognitive outcomes (Leung and 
Kaplan, 2009). Prenatal stress is associated with increased inflammation and cytokine activity, which may also impact child outcomes (Buitelaar et al., 2003). Higher depressive symptoms are associated with less social support (Ren et al., 2018), and decreased social support during pregnancy is associated with lower Apgar scores, worse labor progress, and increased prenatal and postpartum depression (Elsenbruch et al., 2007). Decreased social support and higher maternal depressive symptoms are also associated with a higher risk of developmental delay in children (Huang et al., 2014). It is likely a combination of biological and psychosocial factors that contribute to altered brain development and child outcomes.

This study has some limitations. Because of the timing of recruitment, limited first trimester depressive symptom data were available, and therefore were not analyzed. Greater severity of EPDS depressive symptom scores is associated with a diagnosis of depression, but the EPDS itself is not diagnostic. Therefore, we cannot say whether these women had a clinical diagnosis of depression. The relationships between even mild depressive symptoms and white matter and behavioral changes highlight the importance of even mild depressive symptoms for child outcomes. Furthermore, the EPDS only asks about the preceding $7 \mathrm{~d}$, and thus may not represent symptoms across the entire trimester. The $\mathrm{CBCL}$ is a parent report measure, and therefore may be subject to parental bias and anxiety. Specific to the context of this study, women experiencing more depressive symptoms may answer the CBCL differently from many factors, including attachment and coping. Parental anxiety and social support contribute to child development and may play a role in our sample here. Brain development is known to be nonlinear in children (Reynolds et al., $2019 \mathrm{~b}$ ), although linear fits, as used here, can approximate trends over narrow age ranges. The age range in this study is $2.86-6$ years; and so, it is possible that a linear fit is not ideal. Finally, our sample size was small for mediation, particularly in sex-specific analysis, which results in low power and may increase the FDR (Smaldino and McElreath, 2016). Thus, a significant mediation effect may also be present in girls but was not detected here. Future studies with larger sample sizes and wider age ranges may wish to include nonlinear age terms to better represent brain development.

In conclusion, we show altered structural connectivity of the amygdala in children of mothers with higher prenatal depressive symptoms, and these alterations mediate behavioral dysfunction in boys. These data present evidence of a structural basis for decreased top-down inhibition of the amygdala, resulting in altered behavior in children and may indicate structural abnormalities that predisposes children to develop affective disorders. Indeed, these data may help explain why children born to depressed mothers have a higher risk of developing depression themselves (Pearson et al., 2013). Additionally, we show sexual differentiation, suggesting that males have increased vulnerability to changes in early childhood. These findings suggest a need to improve prenatal care through programs that screen for perinatal depression and address prenatal depression as an important factor in future child health outcomes.

\section{References}

Banks SJ, Eddy KT, Angstadt M, Nathan PJ, Phan KL (2007) Amygdalafrontal connectivity during emotion regulation. Soc Cogn Affect Neurosci 2:303-312.

Barker ED, Jaffee SR, Uher R, Maughan B (2011) The contribution of prenatal and postnatal maternal anxiety and depression to child maladjustment. Depress Anxiety 28:696-702.

Bellani M, Negri GA, Brambilla P (2012) The dysregulation profile in children and adolescents: a potential index for major psychopathology? Epidemiol Psychiatr Sci 21:155-159.
Bergink V, Kooistra L, Lambregtse-van den Berg MP, Wijnen H, Bunevicius R, van Baar A, Pop V (2011) Validation of the Edinburgh Depression Scale during pregnancy. J Psychosom Res 70:385-389.

Bhatia KD, Henderson LA, Hsu E, Yim M (2018) Reduced integrity of the uncinate fasciculus and cingulum in depression: a stem-by-stem analysis. J Affect Disord 235:220-228.

Bjorkquist OA, Olsen EK, Nelson BD, Herbener ES (2016) Altered amygdala-prefrontal connectivity during emotion perception in schizophrenia. Schizophr Res 175:35-41.

Buitelaar JK, Huizink AC, Mulder EJ, de Medina PG, Visser GH (2003) Prenatal stress and cognitive development and temperament in infants. Neurobiol Aging 24:S53-S60.

Burghy CA, Stodola DE, Ruttle PL, Molloy EK, Armstrong JM, Oler JA, Fox ME, Hayes AS, Kalin NH, Essex MJ, Davidson RJ, Birn RM (2012) Developmental pathways to amygdala-prefrontal function and internalizing symptoms in adolescence. Nat Neurosci 15:1736-1741.

Carballedo A, Amico F, Ugwu I, Fagan AJ, Fahey C, Morris D, Meaney JF, Leemans A, Frodl T (2012) Reduced fractional anisotropy in the uncinate fasciculus in patients with major depression carrying the met-allele of the Val66Met brain-derived neurotrophic factor genotype. Am J Med Genet B Neuropsychiatr Genet 159B:537-548.

Chen $\mathrm{CH}$, Suckling $\mathrm{J}$, Ooi $\mathrm{C}$, Fu $\mathrm{CH}$, Williams SC, Walsh $\mathrm{ND}$, Mitterschiffthaler MT, Pich EM, Bullmore E (2008) Functional coupling of the amygdala in depressed patients treated with antidepressant medication. Neuropsychopharmacology 33:1909-1918.

Cheng W, Rolls ET, Qiu J, Xie X, Lyu W, Li Y, Huang CC, Yang AC, Tsai SJ, Lyu F, Zhuang K, Lin CP, Xie P, Feng J (2018) Functional connectivity of the human amygdala in health and in depression. Soc Cogn Affect Neurosci 13:557-568.

Choi J, Jeong B, Rohan ML, Polcari AM, Teicher MH (2009) Preliminary evidence for white matter tract abnormalities in young adults exposed to parental verbal abuse. Biol Psychiatry 65:227-234.

Cox JL, Holden JM, Sagovsky R (1987) Detection of postnatal depression. Br J Psychiatry 150:782-786.

Cullen KR, Westlund MK, Klimes-Dougan B, Mueller BA, Houri A, Eberly LE, Lim KO (2014) Abnormal amygdala resting-state functional connectivity in adolescent depression. JAMA Psychiatry 71:1138-1147.

Davis EP, Hankin BL, Glynn LM, Head K, Kim DJ, Sandman CA (2020) Prenatal maternal stress, child cortical thickness, and adolescent depressive symptoms. Child Dev 91:782-786.

Dawson G, Ashman SB, Panagiotides H, Hessl D, Self J, Yamada E, Embry L (2003) Preschool outcomes of children of depressed mothers: role of maternal behavior, contextual risk, and children's brain activity. Child Dev 74:1158-1175.

De Bellis MD, Keshavan MS, Beers SR, Hall J, Frustaci K, Masalehdan A, Noll J, Boring AM (2001) Sex differences in brain maturation during childhood and adolescence. Cereb Cortex 11:552-557.

Dean DC, Planalp EM, Wooten W, Kecskemeti SR, Adluru N, Schmidt CK, Frye C, Birn RM, Burghy CA, Schmidt NL, Styner MA, Short SJ, Kalin NH, Goldsmith HH, Alexander AL, Davidson RJ, Davidson RJ (2018) Association of prenatal maternal depression and anxiety symptoms with infant white matter microstructure. JAMA Pediatr 172:973-981.

Deng F, Wang Y, Huang H, Niu M, Zhong S, Zhao L, Qi Z, Wu X, Sun Y, Niu C, He Y, Huang L, Huang R (2018) Abnormal segments of right uncinate fasciculus and left anterior thalamic radiation in major and bipolar depression. Prog Neuropsychopharmacol Biol Psychiatry 81:340-349.

Drevets WC, Price JL, Furey ML (2008) Brain structural and functional abnormalities in mood disorders: implications for neurocircuitry models of depression. Brain Struct Funct 213:93-118.

Earls MF, Committee on Psychosocial Aspects of Child and Family Health American Academy of Pediatrics (2010) Incorporating recognition and management of perinatal and postpartum depression into pediatric practice. Pediatrics 126:1032-1039.

El Marroun H, Zou R, Muetzel RL, Jaddoe VW, Verhulst FC, White T, Tiemeier H (2018) Prenatal exposure to maternal and paternal depressive symptoms and white matter microstructure in children. Depress Anxiety 35:321-329.

Elsenbruch S, Benson S, Rücke M, Rose M, Dudenhausen J, PincusKnackstedt MK, Klapp BF, Arck PC (2007) Social support during pregnancy: effects on maternal depressive symptoms, smoking and pregnancy outcome. Hum Reprod 22:869-877. 
Evans J, Melotti R, Heron J, Ramchandani P, Wiles N, Murray L, Stein A (2012) The timing of maternal depressive symptoms and child cognitive development: a longitudinal study. J Child Psychol Psychiatry 53:632640.

Foster CE, Webster MC, Weissman MM, Pilowsky DJ, Wickramaratne PJ, Talati A, Rush AJ, Hughes CW, Garber J, Malloy E, Cerda G, Kornstein SG, Alpert JE, Wisniewski SR, Trivedi MH, Fava M, King CA (2008) Remission of maternal depression: relations to family functioning and youth internalizing and externalizing symptoms. J Clin Child Adolesc Psychol 37:714-724.

Fowler CH, Miernicki ME, Rudolph KD, Telzer EH (2017) Disrupted amygdala-prefrontal connectivity during emotion regulation links stress-reactive rumination and adolescent depressive symptoms. Dev Cogn Neurosci 27:99-106.

Frizzo GB, Pedrini JR, Silva de Souza D, Bandeira DR, Borsa JC (2015) Reliability of Child Behavior Checklist and Teacher's Report Form in a sample of Brazilian children. Universitas Psychol 14:149-156.

Gee DG, Gabard-Durnam LJ, Flannery J, Goff B, Humphreys KL, Telzer EH, Hare TA, Bookheimer SY, Tottenham N (2013) Early developmental emergence of human amygdala-prefrontal connectivity after maternal deprivation. Proc Natl Acad Sci USA 110:15638-15643.

Gerardin P, Wendland J, Bodeau N, Galin A, Bialobos S, Tordjman S, Mazet P, Darbois Y, Nizard J, Dommergues M, Cohen D (2011) Depression during pregnancy: is the developmental impact earlier in boys? A prospective case-control study. J Clin Psychiatry 72:378-387.

Hanson JL, Knodt AR, Brigidi BD, Hariri AR (2015) Lower structural integrity of the uncinate fasciculus is associated with a history of child maltreatment and future psychological vulnerability to stress. Dev Psychopathol 27:1611-1619.

Hay R, Reynolds J, Lebel C (2019) White matter tractography guide. In: Amygdala pathway. Available at https://doi.org/10.6084/m9.figshare. 7865186.v1.

Holmes A, Wellman CL (2009) Stress-induced prefrontal reorganization and executive dysfunction in rodents. Neurosci Biobehav Rev 33:773-783.

Holmes A, Lee PH, Hollinshead MO, Bakst L, Roffman JL, Smoller JW, Buckner RL (2012) Individual differences in amygdala-medial prefrontal anatomy link negative affect, impaired social functioning, and polygenic depression risk. J Neurosci 32:18087-18100.

Holtmann M, Buchmann AF, Esser G, Schmidt MH, Banaschewski T, Laucht M (2011) The Child Behavior Checklist-Dysregulation Profile predicts substance use, suicidality, and functional impairment: a longitudinal analysis. J Child Psychol Psychiatry 52:139-147.

Huang CY, Costeines J, Kaufman JS, Ayala C (2014) Parenting stress, social support, and depression for ethnic minority adolescent mothers: impact on child development. J Child Fam Stud 23:255-262.

Ibrahim K, Eilbott JA, Ventola P, He G, Pelphrey KA, McCarthy G, Sukhodolsky DG (2019) Reduced amygdala-prefrontal functional connectivity in children with autism spectrum disorder and co-occurring disruptive behavior. Biol Psychiatry Cogn Neurosci Neuroimaging 4:10311041.

Jalbrzikowski M, Larsen B, Hallquist MN, Foran W, Calabro F, Luna B (2017) Development of white matter microstructure and intrinsic functional connectivity between the amygdala and ventromedial prefrontal cortex: associations with anxiety and depression. Biol Psychiatry 82:511521.

Jones SL, Dufoix R, Laplante DP, Elgbeili G, Patel R, Chakravarty MM, King S, Pruessner JC (2019) Larger amygdala volume mediates the association between prenatal maternal stress and higher levels of externalizing behaviors: sex specific effects in project ice storm. Front Hum Neurosci 13:144.

Josefsson A, Berg G, Nordin C, Sydsjö G (2001) Prevalence of depressive symptoms in late pregnancy and postpartum. Acta Obstet Gynecol Scand 80:251-255.

Kaplan BJ, Giesbrecht GF, Leung BM, Field CJ, Dewey D, Bell RC, Manca DP, O'Beirne M, Johnston DW, Pop VJ, Singhal N, Gagnon L, Bernier FP, Eliasziw M, McCargar LJ, Kooistra L, Farmer A, Cantell M, Goonewardene L, Casey LM, et al. (2014) The Alberta Pregnancy Outcomes and Nutrition (APrON) cohort study: rationale and methods. Matern Child Nutr 10:44-60.

Kim SJ, Jeong DU, Sim ME, Bae SC, Chung A, Kim MJ, Chang KH, Ryu J, Renshaw PF, Lyoo IK (2006) Asymmetrically altered integrity of cingulum bundle in posttraumatic stress disorder. Neuropsychobiology 54:120-125.
Lautarescu A, Pecheva D, Nosarti C, Nihouarn J, Zhang H, Victor S, Craig M, Edwards AD, Counsell SJ (2020) Maternal prenatal stress is associated with altered uncinate fasciculus microstructure in premature neonates. Biol Psychiatry 87:559-569.

Lebel C, Rasmussen C, Wyper K, Walker L, Andrew G, Yager J, Beaulieu C (2008a) Brain diffusion abnormalities in children with fetal alcohol spectrum disorder. Alcohol Clin Exp Res 32:1732-1740.

Lebel C, Walker L, Leemans A, Phillips L, Beaulieu C (2008b) Microstructural maturation of the human brain from childhood to adulthood. Neuroimage 40:1044-1055.

Lebel C, Walton M, Letourneau N, Giesbrecht GF, Kaplan BJ, Dewey D (2016) Archival report prepartum and postpartum maternal depressive symptoms are related to children's brain structure in preschool. Biol Psychiatry 80:859-868.

Leemans A, Jeurissen B, Sijbers J, Jones DK (2009) ExploreDTI: A Graphical Toolbox for Processing, Analyzing, and Visualizing Diffusion MR Data, Proceedings of the International Society for Magnetic Resonance in Medicine, Honolulu, Hawaii.

Leung BM, Kaplan BJ (2009) Perinatal depression: prevalence, risks, and the nutrition link-a review of the literature. J Am Diet Assoc 109:15661575.

Lohoff FW (2010) Overview of the genetics of major depressive disorder. Curr Psychiatry Rep 12:539-546.

Luking KR, Repovs G, Belden AC, Gaffrey MS, Botteron KN, Luby JL, Barch DM (2011) Functional connectivity of the amygdala in early-childhoodonset depression. J Am Acad Child Adolesc Psychiatry 50:1027-1041.e3.

Malykhin NV, Carter R, Hegadoren KM, Seres P, Coupland NJ (2012) Fronto-limbic volumetric changes in major depressive disorder. J Affect Disord 136:1104-1113.

Marečková K, Klasnja A, Andrýsková L, Brázdil M, Paus T (2019) Developmental origins of depression-related white matter properties: findings from a prenatal birth cohort. Hum Brain Mapp 40:1155-1163.

Merikangas KR, Nakamura EF, Kessler RC (2009) Epidemiology of mental disorders in children and adolescents. Dialogues Clin Neurosci 11:7-20.

Muftuler LT, Davis EP, Buss C, Solodkin A, Su MY, Head KM, Hasso AN, Sandman CA (2012) Development of white matter pathways in typically developing preadolescent children. Brain Res 1466:33-43.

Murray L, Arteche A, Fearon P, Halligan S, Croudace T, Cooper P (2010) The effects of maternal postnatal depression and child sex on academic performance at age 16 years: a developmental approach. J Child Psychol Psychiatry 51:1150-1159.

Noordermeer SD, Luman M, Oosterlaan J (2016) A systematic review and meta-analysis of neuroimaging in oppositional defiant disorder (ODD) and conduct disorder $(\mathrm{CD})$ taking attention-deficit hyperactivity disorder (ADHD) into account. Neuropsychol Rev 26:44-72.

Oberlander TF, Reebye P, Misri S, Papsdorf M, Kim J, Grunau RE (2007) Externalizing and attentional behaviors in children of depressed mothers treated with a selective serotonin reuptake inhibitor antidepressant during pregnancy. Arch Pediatr Adolesc Med 161:22-29.

Ono M, Kikusui T, Sasaki N, Ichikawa M, Mori Y, Murakami-Murofushi K (2008) Early weaning induces anxiety and precocious myelination in the anterior part of the basolateral amygdala of male Balb/c mice. Neuroscience 156:1103-1110.

Park AT, Leonard JA, Saxler PK, Cyr AB, Gabrieli JD, Mackey AP (2018) Amygdala-medial prefrontal cortex connectivity relates to stress and mental health in early childhood. Soc Cogn Affect Neurosci 13:430-439.

Patel TD, Azmitia EC, Zhou FC (1996) Increased 5-HT1A receptor immunoreactivity in the rat hippocampus following 5,7-dihydroxytryptamine lesions in the cingulum bundle and fimbria-fornix. Behav Brain Res 73:319-323.

Pearson RM, Evans J, Kounali D, Lewis G, Heron J, Ramchandani PG, O'Connor TG, Stein A (2013) Maternal depression during pregnancy and the postnatal period. JAMA Psychiatry 70:1312-1319.

Perlman SB, Almeida JR, Kronhaus DM, Versace A, Labarbara EJ, Klein CR, Phillips ML (2012) Amygdala activity and prefrontal cortex-amygdala effective connectivity to emerging emotional faces distinguish remitted and depressed mood states in bipolar disorder. Bipolar Disord 14:162174

Prater KE, Hosanagar A, Klumpp H, Angstadt M, Luan Phan K (2013) Aberrant amygdala-frontal cortex connectivity during perception of fearful faces and at rest in generalized social anxiety discorder. Depress Anxiety 30:234-241. 
Price JL, Drevets WC (2010) Neurocircuitry of mood disorders. Neuropsychopharmacology 35:192-216.

Qiu A, Anh TT, Li Y, Chen H, Broekman BF, Kwek K, Saw S, Chong Y, Gluckman PD, Fortier MV (2015) Prenatal maternal depression alters amygdala functional connectivity in 6-month-old infants. Transl Psychiatry 5:e508.

Rajmohan V, Mohandas E (2007) The limbic system. Indian J Psychiatry 49:132-139.

Ren P, Qin X, Zhang Y, Zhang R (2018) Is social support a cause or consequence of depression? A longitudinal study of adolescents. Front Psychol 9:1634.

Rescorla LA (2005) Assessment of young children using the Achenbach System of Empirically Based Assessment (ASEBA). Ment Retard Dev Disabil Res Rev 11:226-237.

Reynolds J, Grohs M, Lebel C (2019a) White matter tractography guides. FigShare. Available at https://doi.org/10.6084/m9.figshare.7603271.v1.

Reynolds JE, Grohs MN, Dewey D, Lebel C (2019b) Global and regional white matter development in early childhood. Neuroimage 196:49-58.

Reynolds JE, Long X, Paniukov D, Bagshawe M, Lebel C (2020) Calgary Preschool magnetic resonance imaging (MRI) dataset. Data Brief 29:105224.

Rifkin-Graboi A, Bai J, Chen H, Hameed WB, Sim LW, Tint MT, LeutscherBroekman B, Chong YS, Gluckman PD, Fortier MV, Meaney MJ, Qiu A (2013) Prenatal maternal depression associates with microstructure of right amygdala in neonates at birth. Biol Psychiatry 74:837-844.

Rifkin-Graboi A, Meaney MJ, Chen H, Bai J, Hameed WB, Tint MT, Broekman BF, Chong YS, Gluckman PD, Fortier MV, Qiu A (2015) Antenatal maternal anxiety predicts variations in neural structures implicated in anxiety disorders in newborns. J Am Acad Child Adolesc Psychiatry 54:313-321.e2.

Sandman CA, Buss C, Head K, Davis EP (2015) Fetal exposure to maternal depressive symptoms is associated with cortical thickness in late childhood. Biol Psychiatry 77:324-334.

Sheikh HI, Joanisse MF, Mackrell SM, Kryski KR, Smith HJ, Singh SM, Hayden EP (2014) Links between white matter microstructure and cortisol reactivity to stress in early childhood: evidence for moderation by parenting. Neuroimage Clin 6:77-85.
Simmonds DJ, Hallquist MN, Asato M, Luna B (2014) Developmental stages and sex differences of white matter and behavioral development through adolescence: a longitudinal diffusion tensor imaging (DTI) study. Neuroimage 92:356-368.

Smaldino PE, McElreath R (2016) The natural selection of bad science. R Soc Open Sci 3:160384.

Smith SM, Jenkinson M, Johansen-Berg H, Rueckert D, Nichols TE, Mackay CE, Watkins KE, Ciccarelli O, Cader MZ, Matthews PM, Behrens TE (2006) Tract-based spatial statistics: voxelwise analysis of multi-subject diffusion data. Neuroimage 31:1487-1505.

Steffens DC, Taylor WD, Denny KL, Bergman SR, Wang L (2011) Structural integrity of the uncinate fasciculus and resting state functional connectivity of the ventral prefrontal cortex in late life depression. PLoS One 6: e22697.

Taylor AB, MacKinnon DP (2012) Four applications of permutation methods to testing a single-mediator model. Behav Res Methods 44:806-844.

van Eijndhoven P, van Wingen G, van Oijen K, Rijpkema M, Goraj B, Jan Verkes R, Oude Voshaar R, Fernández G, Buitelaar J, Tendolkar I (2009) Amygdala volume marks the acute state in the early course of depression. Biol Psychiatry 65:812-818.

Weinstock M (2005) The potential influence of maternal stress hormones on development and mental health of the offspring. Brain Behav Immun 19:296-308

Weinstock M (2008) The long-term behavioural consequences of prenatal stress. Neurosci Biobehav Rev 32:1073-1086

Wen DJ, Poh JS, Ni SN, Chong YS, Chen H, Kwek K, Shek LP, Gluckman PD, Fortier MV, Meaney MJ, Qiu A (2017) Influences of prenatal and postnatal maternal depression on amygdala volume and microstructure in young children. Transl Psychiatry 7:e1103.

Wong ML, Kling MA, Munson PJ, Listwak S, Licinio J, Prolo P, Karp B, McCutcheon IE, Geracioti TD, DeBellis MD, Rice KC, Goldstein DS, Veldhuis JD, Chrousos GP, Oldfield EH, McCann SM, Gold PW (2000) Pronounced and sustained central hypernoradrenergic function in major depression with melancholic features: relation to hypercortisolism and corticotropin-releasing hormone. Proc Natl Acad Sci USA 97:325-330.

Yoshida S, Oishi K, Faria AV, Mori S (2013) Diffusion tensor imaging of normal brain development. Pediatr Radiol 43:15-27. 